\title{
Beyond Volumes: Extraction of Non-Manifold Topologies from 3D Microscopy Data
}

\author{
Peter Johann Felfer ${ }^{1 *}$
}

1. Department of Materials Science, Institute for General Materials Properties, Friedrich-Alexander Universität Erlangen-Nürnberg, Germany.

* Corresponding author: peter.felfer@fau.de

One of the most interesting uses of 3D high resolution microscopy data is the generation of input data for simulations. However, since all microscopy data inherently contains flaws, it cannot directly be used as simulation input. An approach to allow for so called "experimentally informed simulations" is to use abstractions of 3D microscopy data, upon which simulation volumes can be built up using further knowledge of e.g. the orientation and crystal structure of phases and the atomic structure around crystal defects [1]. One of the main tasks for a new breed of materials scientists, the data scientist is then to extract an abstraction of the crystal defects of a material from the 3D microscopy data.

Traditionally, the 3D analysis of microstructures was limited to the delineation of volumes through scalar properties, possibly combined with the skeletonisation of the defined volumes. The most prominent example of such an analysis is the use of the famous Lorensen-Cline marching cubes algorithm [2]. What this algorithm and in fact all volumetric approaches have in common is that they can only be used to delineate features that constitute a 2 -manifold in $\mathbf{R}^{3}$. While of course in reality any crystal defect occupies a finite volume, this volume may either be beyond the resolution limit of the used microscopy technique or it may correspond well with the conceptual abstraction of the feature. Good examples for this are dislocations, which are abstracted as line like defects, i.e. 1D objects in $\mathbf{R}^{3}$. Other examples are structures that are inherently non-manifold such as grain boundary networks in poly crystals. Here, besides the creation of an abstraction for simulation, also the anlaysis of the network topology can be of great interest.

One of the main reasons that this kind of data abstraction, where objects that are not n- 1 dimensional and non-manifold are created is not as straight forward as the creation of an iso-surface is that there is currently no general algorithm that can provide the topology of the features that are present in the data. In special cases such as point clouds that delineate surfaces, algorithms exist that can be used to create piecewise linear approximations of said surfaces ("surface meshes") [3,4]. To provide a more general framework, the author has developed approaches based on 3D computational geometry that can be used to manually create the topology of the features in the data which is then iteratively fitted to the data [5]. While the main application so far has been the analysis of atom probe tomography data, which exists in the form of 3D point clouds, these approaches are universally usable also for scalar 3D volumes ("voxelisations"). All features are outlined as piecewise linear approximations, this means surfaces as triangle meshes, line-like objects as line segments and singular objects as points. Volumes are implicitly defined by their delineating surfaces and their directionality (surface normal defined by the chirality of the triangle). Since the triangle meshes that make up surfaces and interfaces are made up of edges and vertices, such a description can be used to fully outline any feature in $\mathbf{R}^{3}$, regardless of topology (see. e.g. fig. 1). This includes features of mixed topology with non-manifold sections such as grain boundary networks.

Besides the spatial description of the features for the creation of "digital twins", these abstractions can 
also be used for analysis purposes. The upside of having comprehensive knowledge of the location of the atoms of a material relative to e.g. crystal defects is that predictions of both thermodynamic and kinetic theories can be verified against real data (fig. 2). Such analysis may be the distribution of elements across a moving grain boundary but also the distribution of segregated elements inside a grain boundary, where elements have a much higher mobility compared to the undisturbed crystal volume [6].

\section{References:}

[1] A Prakash et al., Acta Materialia 92(215) (2015), p. 33.

[2] W Lorensen and H Cline, SIGGRAPH Comput. Graph. 21(4) (1987), p. 163.

[3] N Amenta and M Bern, Discrete \& Computational Geometry 22(4) (1999), p. 481.

[4] N Amenta, S Choi and K Ravi, Computational Geometry 19(2-3) (2001), p. 127.

[5] P Felfer and JM Cairney, Ultramicroscopy 169 (2016), p. 62.

[6] The authors acknowledge financial support by the Deutsche Forschungsgemeinschaft (DFG) within the Cluster of Excellence "Engineering of Advanced Materials" (project EXC 315).

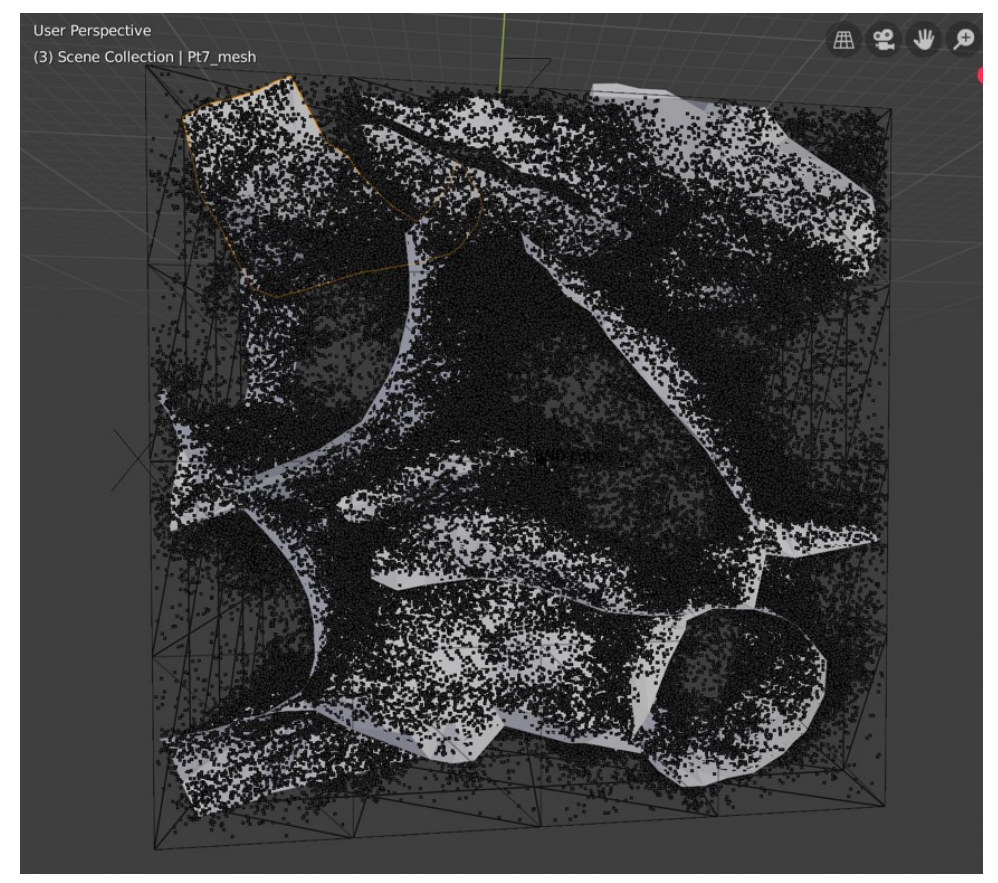

Figure 1. Outline of a complex grain boundary system in an $\mathrm{Al}$ thin film based on atom probe tomography data (Sample courtesy D. Gianola, UCSB, work with J. M. Cairney).
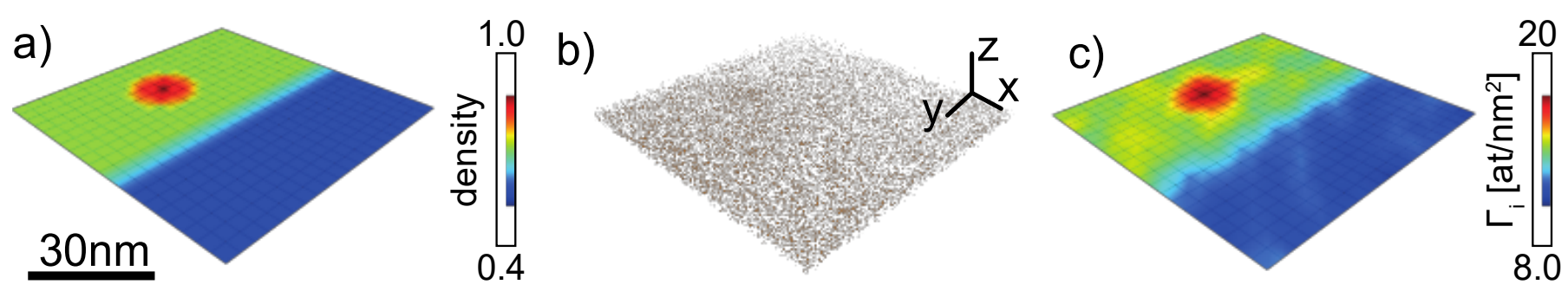

Figure 2. Mapping of the atomic density in a simulated interface for thermodynamic modelling. (Work with J. M. Cairney) 